\title{
The hand radiograph as a diagnostic discriminant between seropositive and seronegative 'rheumatoid arthritis': a controlled study
}

\author{
THOMAS M. BURNS AND ANDREI CALIN \\ From Stanford University and Palo Alto Veterans Hospital, California, USA
}

SUMMARY Although traditional teaching emphasises that $70-80 \%$ of patients with rheumatoid arthritis have positive serological tests for rheumatoid factor, a review of the evidence suggests that the seronegative group has distinctive characteristics. In a blinded and controlled evaluation of hand and wrist films we correctly identified the serological status of 43 out of 46 patients satisfying the ARA criteria for 'definite RA'. The radiographic appearances of the seronegative group differed significantly from those of the seropositive group in (1) degree of juxtalesional osteosclerosis $(p<0.001) ;(2)$ the relative absence of classical subchondral erosions $(p<0.001) ;(3)$ presence of new bone formation $(p<0.001)$; (4) more fusion $(p<0.001) ;(5)$ more asymmetrical joint involvement $(p<0.001)$; and (6) predominant carpal involvement $(p<0.001)$. The nature of the destructive process, as defined radiologically, may be different in patients with seropositive rheumatoid arthritis from that seen in individuals with so-called 'seronegative rheumatoid arthritis'.

The discovery of serum rheumatoid factor(s) and HLA B27 has done much to further our understanding of the nosology of the inflammatory arthropathies. The B27. related spondylarthropathies are now known to be distinct entities, and the old term rheumatoid variants is no longer applicable. However, there is still confusion about the nature of another seronegative group, 'seronegative RA'. ${ }^{1}$

Table 1 outlines the classification of inflammatory arthropathies of unknown aetiology. These can be conveniently subdivided into 2 groups-the seropositive and seronegative. The division is somewhat arbitrary in that some diseases, for example, systemic lupus erythematosus (SLE), can be either seropositive or seronegative for rhe umatoid factor. However, concomitant clinical findings aid in making these diagnoses. Moreover, serological tests can convert at a time distant from the initial presentation, so that some patients with so-called 'seronegative rheumatoid arthritis' may later be classified as having seropositive disease, and conversely the serological status can change from positive to negative with drug therapy ${ }^{23}$ and perhaps time. Earlier studies suggest that this crossover occurs on the order of $12-15 \% .^{4}$

Accepted for publication 11 January 1983.

Correspondence to Dr A. Calin, Room S102A, Division of Immunology, Stanford University Medical Center, Stanford, California 94305, USA.
A major difficulty in diagnosis is represented by those patients with a persistently seronegative inflammatory arthropathy who have neither overt coexistent disease like psoriasis or inflammatory bowel disease nor a B27 related spondylarthropathy. These seronegative patients virtually never develop the extra-articular manifestations classically seen in seropositive rhe umatoid disease-for example, subcutaneous nodules, pleuropericarditis, Felty's syndrome, vasculitis, sicca syndrome, or interstitial lung disease..$^{5}$ In part the difficulty in diagnosis has been fostered by the general application of the ARA criteria for rheumatoid arthritis, ${ }^{6}$ which were intended for epidemiological research. Patients with almost any inflammatory arthropathy, regardless of serological status, can easily meet 5 out of 11 criteria, the reby yielding a diagnosis of 'definite' rheumatoid arthritis. In 1960 Dixon $^{7}$ reported a follow-up of 63 patients who were consistently seronegative and in whom no diagnosis other than rheumatoid arthritis (or polyarthritis of undetermined type) could be made by accepted criteria. Twelve of 63 patients converted to seropositivity and were felt to have typical seropositive rheumatoid arthritis. The other patients belonged to a heterogeneous group of disorders which led him to conclude: 'A reproducible diagnosis of rhe umatoid arthritis requires a positive specific SCAT as well as clinical evidence of poly- 
Table 1 Major adult arthropathies of unknown aetiology according to serological status

\begin{tabular}{|c|c|}
\hline Sero (+) & Sero (-) \\
\hline 1. Rheumatoid arthritis & $\begin{array}{l}\text { 1. Spondylarthropathies (AS, RD, PsA, Ar with IBD) } \\
\text { with co-disease clinically present }\end{array}$ \\
\hline $\begin{array}{l}\text { 2. 'Collagen vascular' disease } \\
\text { (SLE, PSS, DM/PM, MCTD) }\end{array}$ & $\begin{array}{l}\text { 2. Spondylarthropathies with co-disease destined to develop later } \\
\text { 3. Polyarthritis with development of seropositivity later } \\
\text { 4. Remaining seronegative without development of GU, } \\
\text { spinal, skin, or bowel co-disease }\end{array}$ \\
\hline
\end{tabular}

SLE = systemic lupus erythematosus; $P S S=$ progressive systemic sclerosis; DM/PM=dermato/polymyositis; $M C T D=$ mixed connective tissue disease; $A S=$ ankylosing spondylitis; $R D=$ Reiter's disease; $P s A=$ psoriatic arthritis; $I B D=$ inflammatory bowel disease; $G U=g e n i t o u r i n a r y ;$ $\mathrm{Ar}=$ arthritis.

arthritis. In clinical studies of this disease, the results of agglutination tests should be stated, and patients with persistently negative tests excluded or separately assessed.'

Unfortunately many recent investigators have failed to heed Dixon's advice and have treated rheumatoid arthritis patients as a homogeneous group. Hence basic questions are still unanswerable. For example, are gold and D-penicillamine more efficacious and/or less toxic in seropositive than seronegative patients? Some authors would argue that both gold and D-penicillamine are more nephrotoxic in seronegative patients, ${ }^{89}$ but larger studies are needed to assess their results. Do these seronegative patients respond to indomethacin and phenylbutazone similarly to the seronegative spondylarthritis group? Do immunosuppressives have a more important role in this disorder? Are underlying pathogenetic mechanisms the same? Recent work has suggested that seronegative mononuclear leucocytes behave differently than seropositive mononuclear leucocytes. ${ }^{10}$

Another barrier to accurate diagnosis has been the often claimed clinical statement that 'seronegative rhe umatoid arthritis' is only a mild subset of seropositive disease. The accuracy of this statement is hard to verify given that 'seronegative rheumatoid arthritis' has included a heterogeneous population. Earlier studies report equally destructive disease in some patients with negative serological tests, ${ }^{7}$ while others have reported the occurrence of more complete fusion $^{11}$ in the seronegative population. Certainly seronegative disease encompasses a spectrum of severity of joint disease which can parallel that of seropositive disease.

This study is an attempt to define the nature of the radiological change in patients with 'seronegative rhe umatoid arthritis'. Patients with seropositive disease, matched for age, sex, and disease duration, were used as controls.

If radiographic differences were found to be predictive of the serological status, it would suggest that there may be different pathological processes in the 2 diseases. Seropositive rheumatoid arthritis is radiographically characterised by juxta-articular osteoporosis, symmetrical involvement of carpal, metacarpophalangeal (MCP), and proximal interphalangeal (PIP) joints, destruction by classical, subchondral erosions beginning at the insertion of the joint capsule, less fusion, and lack of reactive new bone formation. Seronegative disease should be characterised by the opposite qualities if it is distinctive.

\section{Materials and methods}

All 84 patients diagnosed as having persistently 'seronegative rheumatoid arthritis' at the Stanford University Medical Center and Palo Alto VA hospital clinics were reviewed. Seronegative patients with spondylarthritis, psoriasis, inflammatory bowel disease, or adult onset Still's disease were excluded. All 34 patients with hand radiographs were selected. Five of 34 had to be excluded because of an inability to find appropriately matched seropositive controls with radiographs. An attempt was made to match each of the 29 seronegative subjects with 2 seropositive patients from the same clinic controlled for age, sex, and disease duration. Only 41 of a total of 233 consecutive seropositive (latex $>1: 80$ ) individuals were approprate (i.e., 12 of the 29 had 2 seropositive controls). A similar proportion of the seropositive patients had had hand radiographs performed. The mean ages were 53 and 55 years respectively in each group, the female to male ratio was 5:1, and the disease duration was 8.9 and 8.8 years respectively.

Initially, all films were evaluated without knowledge of the patient's identity or serological status (all markings were obscured) by a disease severity index scored on a scale of $0-3$, where $0=$ normal, $1=$ mild, 2 =moderate, $3=$ severe radiological destruction. Thereafter the films were analysed for the presence or absence of osteosclerosis, classical erosions, global symmetry, new bone formation, fusion, and pre- 
dominance of carpal involvement. For the purposes of standardisation a classical erosion was defined as a defect of the articulating surface of subchondral bone with a clear destruction of the cortical margin and an osteoporotic base. Finally, a prediction as to serological status was made on the basis of this composite analysis. Significance was calculated by using either a chi-square test for goodness of fit or a chi-square contingency table with Yates's correction.

\section{Results}

An average destructive index was calculated for each group by adding all individual scores and dividing by the total number of films in each group. The films from seronegative patients (hereafter referred to as seronegative films) had an average destructive score of 1.62 , while the films from seropositive patients (seropositive films) averaged 1.61. As seen in Table 2 , these scores reflected noticeable destructive change in 16 of 29 seronegative films and 30 of 41 seropositive films. The remainder of the films were graded as 0 . Thus the degree of radiological destruction in these patients with previous hand radiographs was comparable with both mild and severe disease occurring in both groups.

Table 3 gives the percentages of the 16 seronegative and the 30 seropositive patients who had the 6 radiographic qualities listed above. The remaining 13
Table 2 The grade and degree of destruction and correct blinded readings of serological status among the 29 seronegative and 41 seropositive radiographs

\begin{tabular}{lcll}
\hline & Sero (-) & Sero (+) & Total \\
\hline Films & 29 & 41 & 70 \\
Destruction (grade 0-3) & 1.62 & 1.61 & \\
Destruction (no.) & 16 & 30 & 46 \\
Correct blind reading & 14 & 29 & $43(\mathrm{p}<0 \cdot 001)$ \\
\hline
\end{tabular}

Table 3 The nature of radiographic damage among those 46 films (16 seronegative, 30 seropositive) showing evidence of destructive change

\begin{tabular}{lccc}
\hline & $\begin{array}{l}\text { Sero (-) } \\
(n=16)\end{array}$ & vs. $\begin{array}{l}\text { Sero (+) } \\
(n=30)\end{array}$ & \\
\hline Osteosclerosis & $87 \%$ & $11 \%$ & $\mathrm{p}<0.001$ \\
Classical erosions* & $12 \%$ & $93 \%$ & $\mathrm{p}<0.001$ \\
Global symmetry ${ }^{\dagger}$ & $6 \%$ & $93 \%$ & $\mathrm{p}<0.001$ \\
New bone formation & $69 \%$ & $7 \%$ & $\mathrm{p}<0.001$ \\
Fusion & $94 \%$ & $30 \%$ & $\mathrm{p}<0.001$ \\
Carpal predominance & $88 \%$ & $7 \%$ & $\mathrm{p}<0.001$ \\
\hline *Erosions: number per & & & \\
\multicolumn{1}{c}{ patient } & 0.3 & 12 & \\
+ Wrist alone: & $6 / 12$ & $15 / 17$ &
\end{tabular}

and 11 films, respectively, were normal and are not commented on further.

Juxta-articular osteosclerosis occurred predominantly in the seronegative state $(87 \%$ versus $11 \%$,

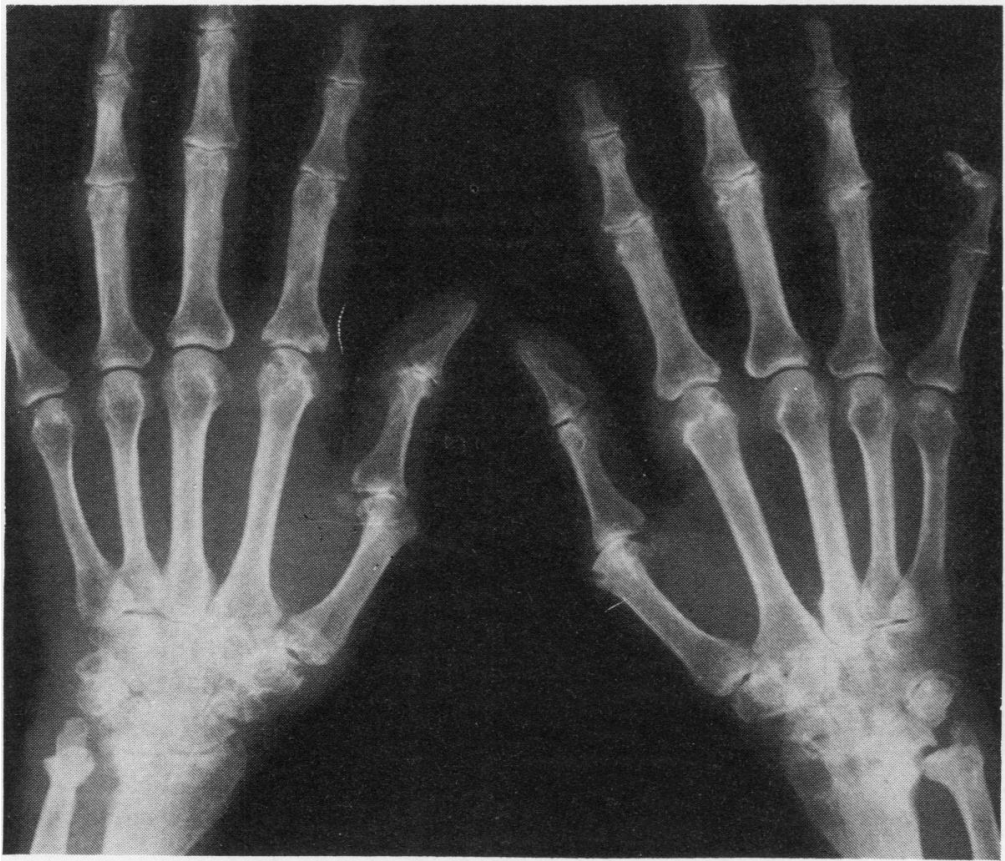

Fig. 1A Hand radiograph of 61-year-old woman who has seropositive rheumatoid arthritis of 15 years, duration. Note. symmetrical wrist and $\ddot{M} C P$ involvement with many classical erosions. Both osteoporosis and osteosclerosis are present at the MCPs and wrists, respectively. 
$\mathrm{p}<0.001)$. Even though sclerosis has been stated to occur as a late sequela of any destructive process, the disease-duration-matched seropositive films showed predominant osteoporosis juxta-articularly.

Classical erosions occurred in only 2 patients $(12 \%)$ with seronegative disease, while it was a hallmark in $93 \%$ of seropositive cases $(p<0 \cdot 001)$. Moreover, even when they did occur, the average number of erosions per patient was only $0.3 \mathrm{com}$ pared with 12 in the seropositive patients. Cysts and geodes were seen in both seronegative and seropositive films, but since there was no loss of cortex they were not considered as 'classical erosions'.

Global symmetry refers to the overall pattern of affected joints. If all affected joints were involved symmetrically, the film was graded as being globally symmetrical. This term does not necessarily signify equally destructive disease in each joint pair but only the presence or absence of radiological change. Even though the seronegative group had symmetrical wrist

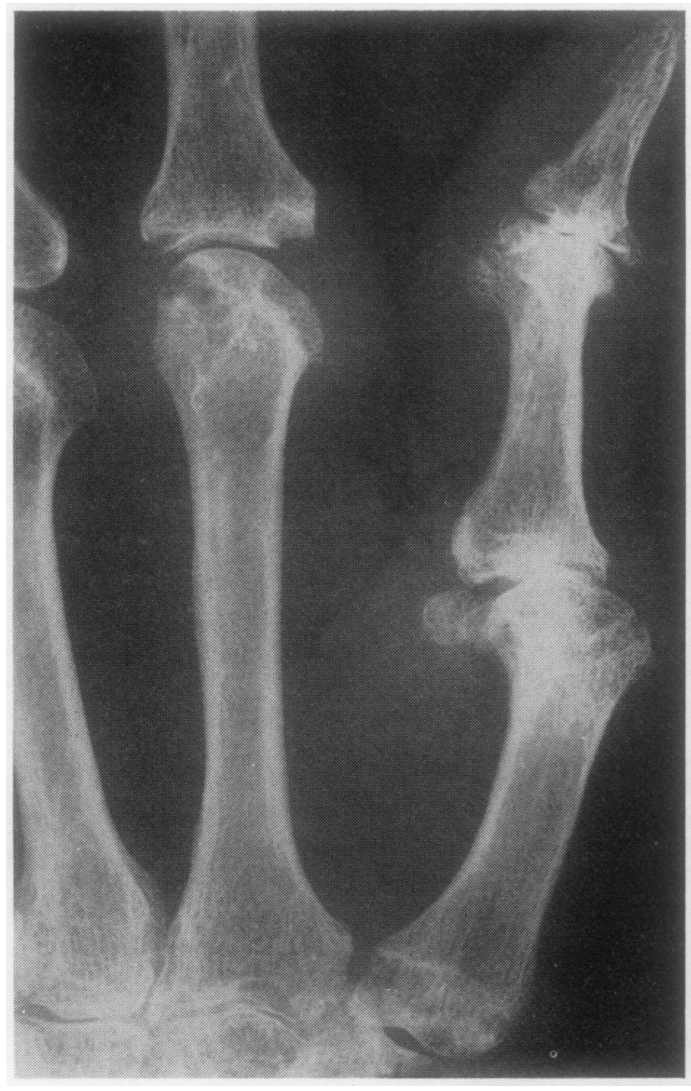

Fig. 1B Close-up of Fig. 1a to demonstrate clearly marginated cortical erosions beginning at joint capsule insertion sites.

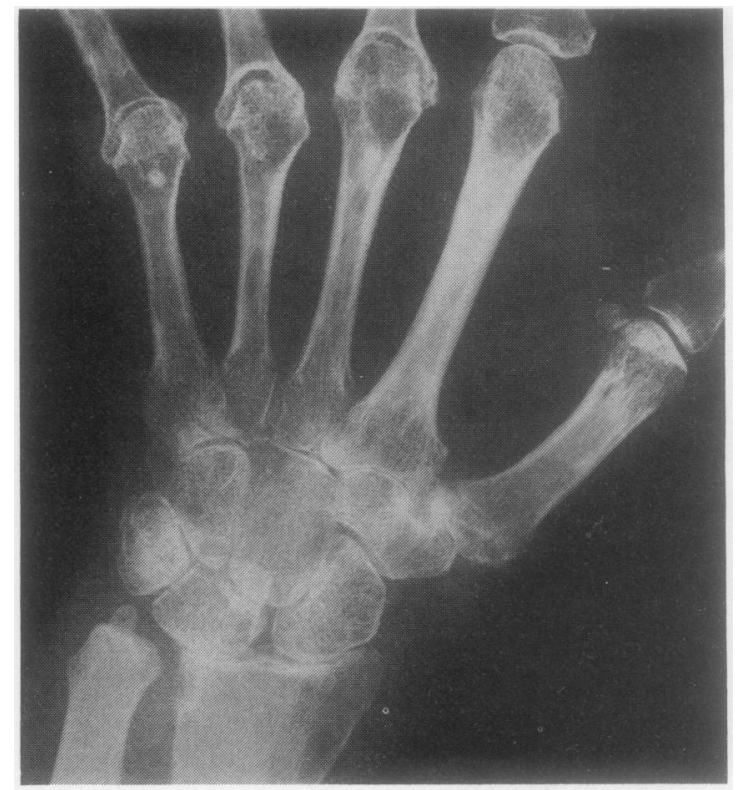

Fig. 2A Left hand radiographs of 52-year-old woman with history of 'seronegative rheumatoid arthritis' of 8 years' duration. Note loss of cartilage space with absence of classical erosions at 3rd, 4th, and 5th MCP joints. The only erosion seen is at the inferior radioulnar joint, and it is surrounded by a sclerotic rim of new bone.

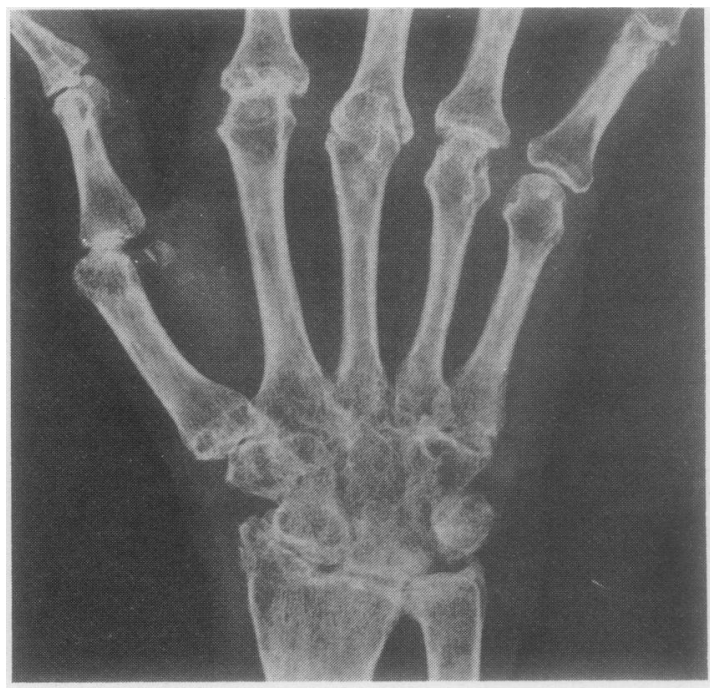

Fig. 2B Same patient as in Fig. 2A showing right hand. Note asymmetrical wrist and MCP disease with partial fusion at the carpal-metacarpal and intracarpal joints. 
involvement in 6 out of 12 cases, only $6 \%$ of the total seronegative group had all joint pairs symmetrically involved compared with $93 \%$ of the seropositive group $(\mathrm{p}<0 \cdot 001)$.

The juxta-articular new bone formation characteristically occurred at the inferior radioulnar joint, although it was also seen at the MCP and distal interphalangeal (DIP) joints. Whereas it occurred in 11 of 16 seronegative patients $(69 \%)$, it appeared in only 2 of 30 seropositive patients.

Both complete and incomplete ankylosis occurred more frequently in seronegative cases $(94 \%)$ than seropositive ones $(30 \%)(p<0 \cdot 001)$. Again the wrist was the most common region affected.

Carpal predominance refers to the fact that the involvement was maximal at that location. When the carpus was involved in seropositive disease, it was almost universally accompanied by MCP and/or PIP involvement, so that in only 2 out of $30(7 \%)$ patients was the carpal involvement predominant. This figure compares with $88 \%$ carpal predominance in the seronegative condition $(p<0 \cdot 001)$.

Fig. $1 \mathrm{~A}$ is an example of a seropositive patient with symmetrical MCP and wrist involvement and multiple classical erosions. Even though this film shows both osteoporosis at the MCPs and osteosclerosis at the wrists, the symmetry and the presence of multiple classical erosions are typical of seropositivity.

Fig. $1 \mathrm{~B}$ is an enlargement of the left first and second digits from the patient in Fig. 1A. It shows the cortical defects of classical erosions seen especially at the capsular insertion sites.

By contrast, joint destruction in the absence of classical erosions was the rule in $88 \%$ of seronegative patients. Fig. 2A shows clear-cut obliteration of the joint space due to cartilage destruction but without the well-delineated erosions so typical of seropositive disease. Note that the only clear erosion is seen at the left inferior radioulnar joint and that it has a sclerotic border of fine new bone surrounding it. Fig. $2 \mathrm{~B}$ is the right hand of the same seronegative patient showing asymmetrical wrist and MCP involvement as well as a marked degree of carpal fusion.

Figs. $3 A$ and $3 B$ show reactive new bone formation at the right inferior radioulnar joint and the right first $\mathrm{MCP}$, as well as the asymmetrical pattern of destruction without erosions at the MCP joints. The wrists are symmetrically and predominantly involved, with almost total fusion bilaterally. There is also juxtaarticular sclerosis at the radiocarpal and MCP joints. Again there may be a single erosion at the distal end of the right radius, but this contrasts with an average of 12 erosions per film in the seropositive patients.

Fig. $4 \mathrm{~A}$ is a close-up view of the right hand from another seronegative patient. It demonstrates the destructive carpal involvement with partial fusion

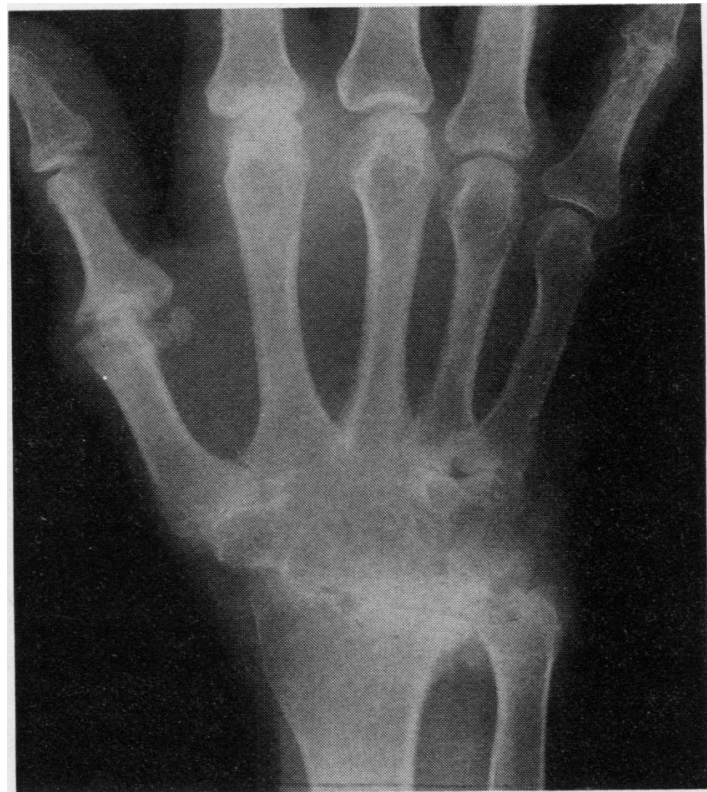

Fig. 3A

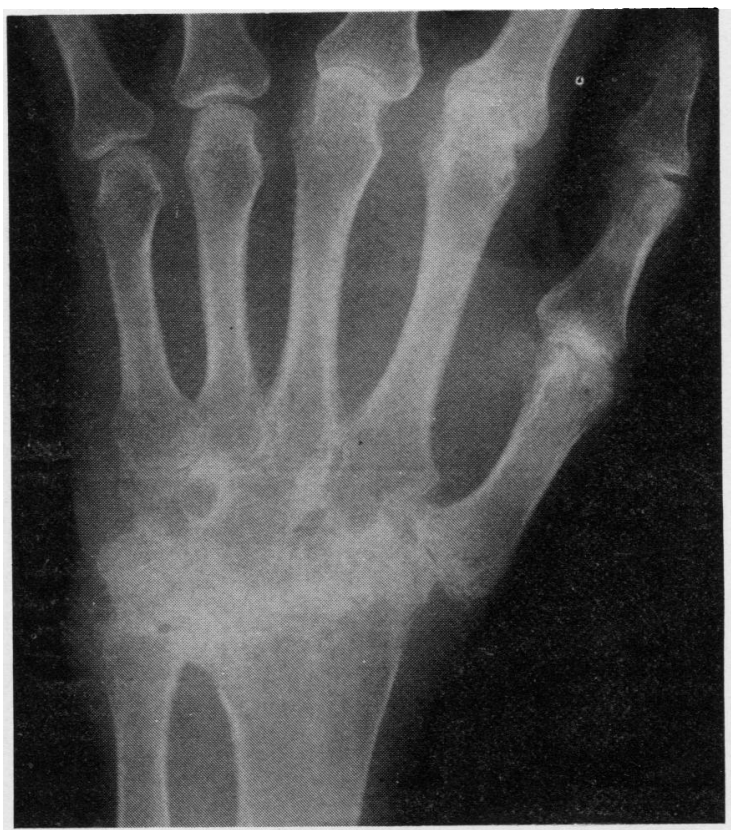

Fig. 3B

Figs. 3A and B Hand radiographs of 35-year-old male with a 13-year history of 'seronegative rheumatoid arthritis.' Note bilateral carpal predominance with almost total carpal fusion. There is also new bone formation at the right radioulnar joint and right first MCP joint as well as a juxta-articular osteosclerosis at the radiocarpal and $M C P$ joints. 


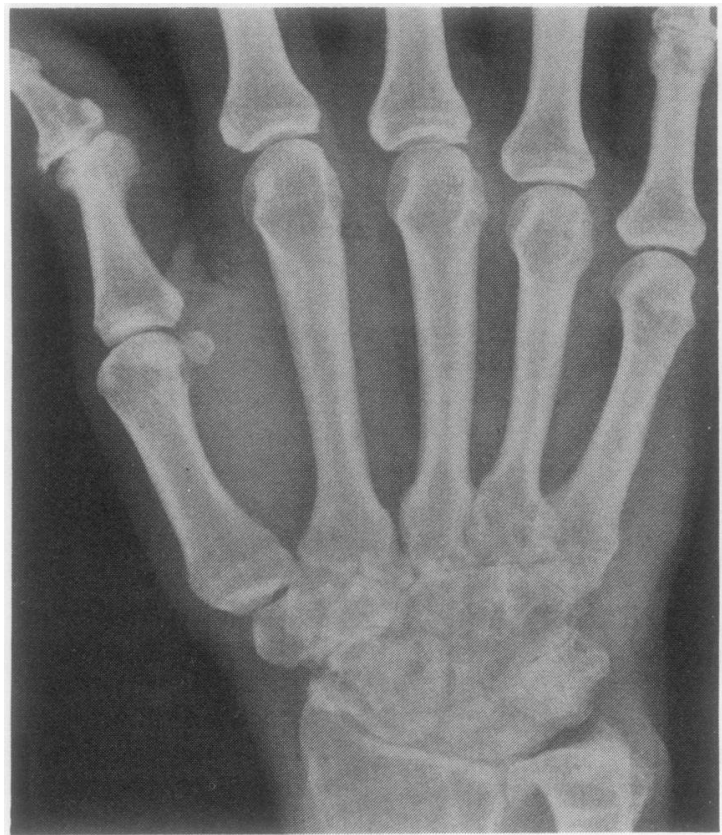

Fig. 4A Hand radiograph of 56-year-old female with 14-year history of 'seronegative rheumatoid arthritis'. Note destruction and partial fusion of the right wrist with new bone formation at the radioulnar joint.

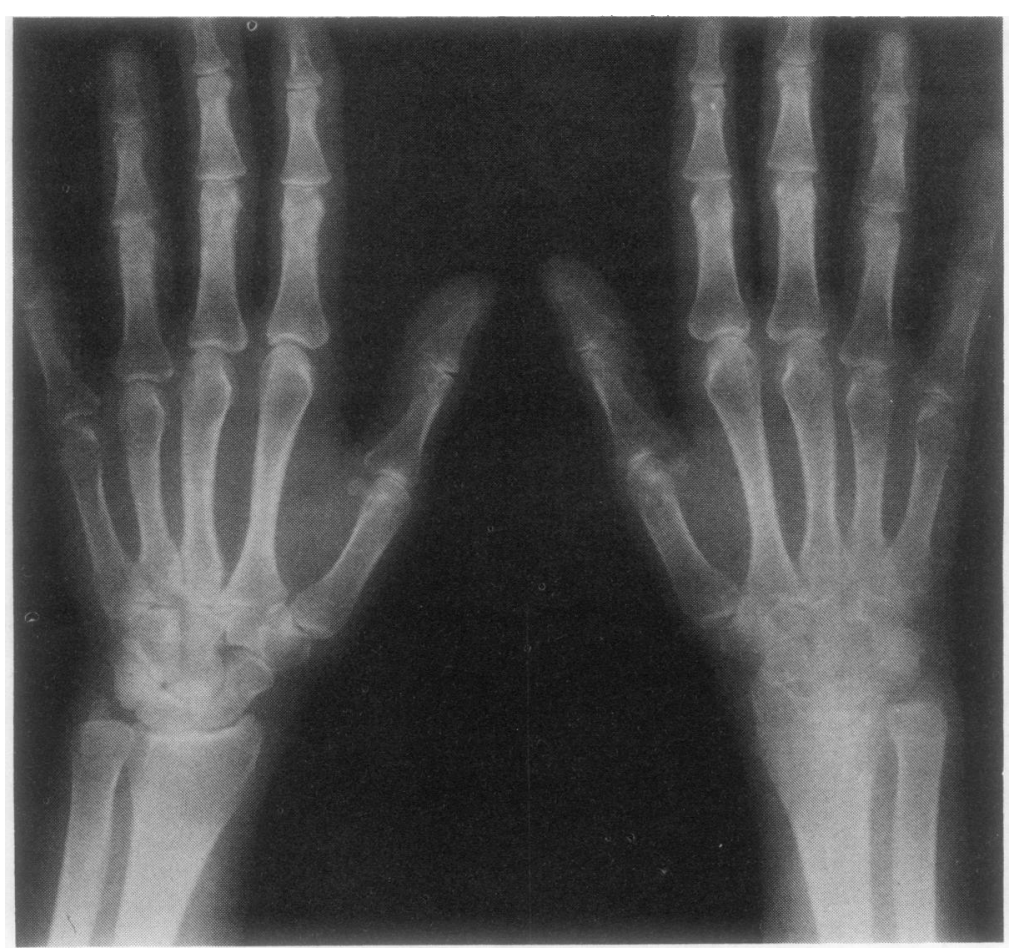

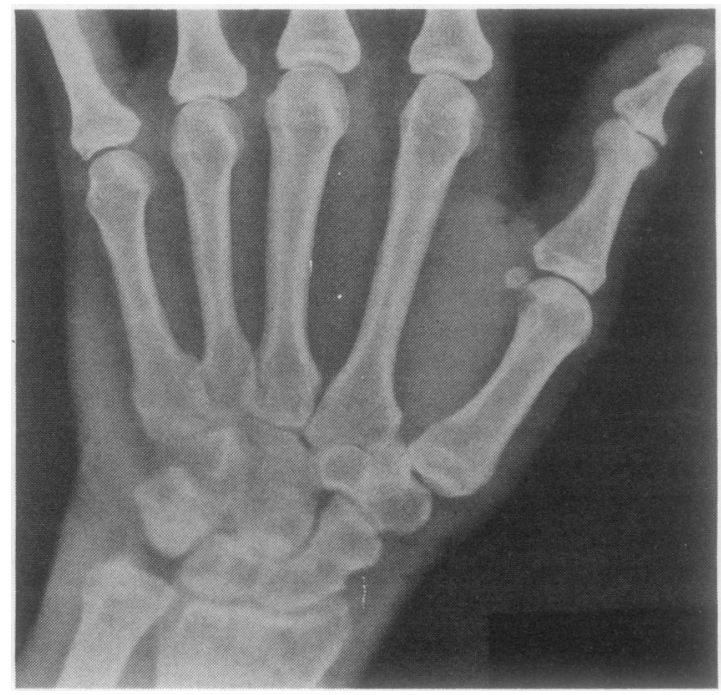

Fig. 4B Contralateral wrist is normal.

Fig. 5 Fifty-year-old female with a 6-year history of 'seronegative rheumatoid arthritis'. Again note predominant asymmetrical wrist involvement with partial fusion. 
and reactive new bone formation at the inferior radioulnar joint. The left wrist (Fig. 4B) is radiologically intact, emphasising the asymmetry at the wrists.

Fig. 5 is similar to Figs. $4 \mathrm{~A}, \mathrm{~B}$ in that it shows predominant carpal involvement that is asymmetrical. Note the absence of classical well-marginated erosions and the partial fusion of the right intracarpal bones.

\section{Discussion}

One of the most important pre requisites to performing comparative studies is to ensure the homogeneity of the patient population under consideration. Most modern studies of rheumatoid arthritis include patients with either definite or classical disease, but rarely are the results analysed according to the serological status. As we have argued elsewhere, ${ }^{1}$ most 'seronegative rheumatoid arthritis' patients can meet only 5 ARA criteria, since nodules, erosion, and seropositivity are usually interdependent. Therefore these 'definite rheumatoid arthritis' patients may indeed be quite different from those patients with classical disease.

A review of the earlier literature corroborates the notion that persistently 'seronegative rheumatoid arthritis' may be distinct from seropositive disease. As early as 1959 Kellgren and Ball ${ }^{12}$ reported on the clinical significance of a positive rhe umatoid factor as determined by the sheep cell agglutination test (SCAT). By carefully re-evaluating the diagnoses of those patients with persistently seronegative disease and excluding others as alternate diagnoses became evident, they found that $96 \%$ of males and $92 \%$ of females with definite rheumatoid arthritis were seropositive, percentages which are higher than the often quoted $70-80 \%$ figure..$^{13} 14$

Early family data ${ }^{15}{ }^{16}$ show that definite or classical rhe umatoid arthritis occurs significantly more frequently in relatives of seropositive probands. By contrast, 'seronegative rhe umatoid arthritis' appears not to be familial. There are equivalent data from twin studies showing concordance of disease only in the seropositive disorder. ${ }^{16}$

Recent immunogenetic data may provide a clue to this familial clustering. HLA DR4 has been associated with seropositive rheumatoid arthritis in $50-60 \%$ of cases. ${ }^{17}{ }^{18}$ However, the frequency of DR4 in 'seronegative rheumatoid arthritis' patients is no different from that in control populations ${ }^{18-20}$ in studies involving larger numbers of patients and appropriate control groups. Conflicting results have been noted by 2 groups of investigators. ${ }^{2122}$

In addition to these epidemiological and genetic data many papers ${ }^{11} 152324$ describe clinical differences between seropositive disease and 'seronegative rhe umatoid arthritis'. Clearly nodules, erosions, sicca syndrome, Felty's syndrome, vasculitis, pleuro- or pericarditis, and interstitial lung disease occur almost exclusively in seropositive patients. ${ }^{25}$ Although some authors would argue that the lack of these extraarticular manifestations signifies a milder form of disease, an equally acceptable interpretation is that the diseases are indeed distinct.

It is of interest, however, that only a few references could be found on the radiographic traits of seronegative patients. Bland and Brown ${ }^{15}$ report that 'radiologic rheumatoid arthritis occurred significantly more frequently in the MCP and MTP joints in the seropositive than in the seronegative group', but fail to define what they meant by 'radiologic rheumatoid arthritis'. Lawrence ${ }^{16}$ remarks that 'radiological evidence of grade 3-4 erosive arthritis of the hands and feet' occurred 6.8 times more frequently than expected in first-degree relatives of seropositive rhe umatoid arthritis probands as compared with only 1.2 times more frequently than expected in the relatives of 'seronegative rhe umatoid arthritis' probands. Sharp et al. ${ }^{26}$ reported that the titre of rheumatoid factor was positively related to the rate of progression of joint defects on the radiograph, but the study included only 6 patients with a negative rheumatoid factor. It was with this scarce background that we undertook the current controlled study in an attempt to define the actual destructive nature of 'seronegative rheumatoid arthritis' and to compare it with that seen in seropositive disease.

Our first finding, that the seronegative group had an equivalent degree of destruction as had the seropositive group, must be interpreted cautiously. This observation cannot be extended to include the whole population of 'seronegative rheumatoid arthritis' patients. Our study group included those patients who had noticeable destructive changes on the hand and wrist radiographs and their matched seropositive controls. It is still not clear what percentage of seronegative patients will go on to develop destructive changes or to what extent changes at the hand and wrist mirror those occurring elsewhere. However, our results do confirm earlier work by Dixon $^{7}$ in which the 'seronegative rheumatoid arthritis' group had a range from mild to severe destructive disease.

The most important point is that, when there was radiographic change, seronegative disease could be distinguished from seropositive disease on the basis of 6 radiographic criteria. These differences suggest that a different pathogenetic mechanism may underly seronegative arthritis. Some radiographic changes are reminiscent of those seen in psoriatic arthritis; however, none of these patients had psoriasis for an average duration of 8.9 years. It is therefore difficult 
to conceptualise this group of patients as psoriatic arthritis sine psoriasis.

An interesting parallel might be drawn with the $30-40 \%$ of patients with juvenile chronic arthritis who are seronegative and DR4 negative. Could our 'seronegative rheumatoid arthritis' subset be the adult equivalent of this juvenile group? Unfortunately no long-term follow-up radiographic studies have been done on this subset of JCA patients to serve as a comparison group. Certain features in adult-onset Still's disease ${ }^{27}$ mimic those seen in our patients.

Until further comparative studies can be performed, we consider that the term seronegative polyarthritis should be used in place of 'seronegative rheumatoid arthritis'. This substitution will allow accurate long-term follow-up of this group of patients with regard to differential response to therapy, prognosis, and incidence of side effects. It will also facilitate more accurate basic research into the underlying pathogenesis of joint destruction, since the investigators will focus on patients who clearly have seropositive rheumatoid arthritis and separately on individuals who have seronegative disease, the majority of whom may not have 'seronegative definite rheumatoid arthritis'.

We gratefully acknowledge the special contribution of Doni Saunders in the preparation of this manuscript.

\section{References}

1 Calin A, Marks S. The case against seronegative rheumatoid arthritis. Am J Med 1981; 70: 992-4.

2 Gottlieb N. Chrysotherapy. Bull Rheum Dis 1976-7; 27: 912-7.

3 Jaffe I. The effect of penicillamine on the laboratory parameters in rheumatoid arthritis. Arthritis Rheum 1965; 8: 1064-79.

4 Wright V, Moll J M H. Seronegative polyarthritis. Amsterdam: Elsevier/North Holland Biomedical Press, 1976: 397-410.

5 Decker J L, Plotz P M. Extra-articular rheumatoid disease. In: McCarty D J, ed. Arthritis and allied conditions. Ninth ed. Philadelphia: Lea and Febiger, 1979: 470-90.

6 Ropes M W, Bennet G A, Cobb S, Jacox R, Jessar R A. 1958 Revision of diagnostic criteria for rheumatoid arthritis. Bull Rheum Dis 1959; 9: 175-6.

7 Dixon A St J. 'Rheumatoid arthritis' with negative serological reaction. Ann Rheum Dis 1960; 19: 209-17.
8 Skrifvars B V, Tornroth T S, Talliqvist G N. Gold-induced immune complex nephritis in seronegative rheumatoid arthritis. Ann Rheum Dis 1977; 36: 549-66.

9 Corke C F, Huskisson E. Factors affecting the development of penicillamine side-effects. Rheumatol Rehabil 1978; 17: 34-7.

10 Alarcon G S, Koopman W F, Schrohenloher R E. Differential patterns of in vitro IgM rheumatoid factor synthesis in seronegative and seropositive rheumatoid arthritis. Arthritis Rheum 1982; 25: 150-5.

11 Mongan E S, Atwater E C. A comparison of patients with seronegative and seropositive rheumatoid arthritis. Med Clin North Am 1968; 52: 533-8.

12 Kellgren J H, Ball J. Clinical significance of the rheumatoid serum factor. $\mathrm{Br} M e d \mathrm{~J}$ 1959; i: 523-31.

13 Davis J S. Rheumatoid factors. In: Cohen A J, ed. Rheumatology and immunology. New York: Grune and Stratton, 1979: 77-80.

14 Ball J, Lawrence J S. Epidemiology of the sheep cell agglutination test. Ann Rheum Dis 1961; 20: 235-43.

15 Bland J H, Brown E W. Seronegative rheumatoid arthritis: clinical, radiological and biochemical differences. Ann Intern Med 1964; 60: 88-94.

16 Lawrence J S. Heberden oration, 1969: Rheumatoid arthritis: nature or nurture? Ann Rheum Dis 1970; 29: 357-79.

17 Dobloug J H, Forre O, Kass E, Thorsby E. HLA Antigens and rheumatoid arthritis. Arthritis Rheum 1980; 23: 309-13.

18 Stastny P. Rheumatoid arthritis. Joint report from the VIIIth International Histocompatibility Workshop, Los Angeles. 1980: 681-6.

19 Espinoza L R, Vasey F B, Gaylord S W, et al. Histocompatibility typing in the seronegative spondyloarthropathies: a survey. Semin Arthritis Rheum 1982; 11: 375-81.

20 Alarcon G S, Koopman W J, Acton R T, Barger B O. Seronegative rheumatoid arthritis: a distinct immunogenetic disease? Arthritis Rheum 1982; 25: 502-7.

21 Panayi G S, Wooley P H, Batchelor J R. HLA-DRw4 and rheumatoid arthritis. Lancet 1979; i: 730.

22 Scherak O, Smolen J S, Mayr W R. Rheumatoid arthritis and B lymphocyte alloantigen HLA-DRw4. J Rheumatol 1980; 7: 9-12.

23 Mongan E S, Cass R M, Jacox R F. A study of the relation of seronegative and seropositive rheumatoid arthritis to each other and to necrotizing vasculitis. Am J Med 1969; 47: 23-35.

24 Cats A, Hazevoet H M. Significance of positive tests for rheumatoid factor in the prognosis of rheumatoid arthritis. Ann Rheum Dis 1969; 29: 254-60.

25 Gordon D, Stein J L, Broder I. The Extra-articular features of rheumatoid arthritis. Am J Med 1973; 54: 445-52.

26 Sharp J T, Lidsky M D, Collins L C, Moreland J. Methods of scoring the progression of radiologic changes in rheumatoid arthritis: correlation of radiologic, clinical and laboratory abnormalities. Arthritis Rheum 1971; 14: 706-20.

27 Elkon K B, Hughes G R V, Bywaters E G L, et al. Adult-onset Still's disease: twenty-year follow-up and further studies of patients with active disease. Arthritis Rheum 1982; 25: 647-54. 